\title{
Um Mapeamento de Ferramentas Digitais para Criação de Narrativas Não-Lineares
}

\section{Bruno Morais Neves de Castro ${ }^{1}$, Matheus Breno Batista dos Santos ${ }^{1}$, Jaline Gonçalves Mombach ${ }^{1}$}

\author{
${ }^{1}$ Instituto de Educação, Ciência e Tecnologia de Brasília - Campus Brasília \\ Setor de Grandes Áreas Norte 610 - Asa Norte - 70830-450 - Brasília - DF - Brasil \\ \{brunomoraisnc, mths.brn@gmail.com\}@gmail.com \\ jaline.mombacheifb.edu.br
}

\begin{abstract}
Nonlinear narratives allow the user to guide the flow of their story, and they can follow different paths as the storyline evolves, exploring different levels of logic and creativity. However, digital tools for this creation are unknown to many educators. Therefore, this paper aims to present a mapping of works that explore nonlinear narratives in the Brazilian context of Computers in Education. Systematic mapping was the method adopted, including gray literature. Results indicate that publications on this subject there is a small number. Also, there are limitations in existing tools on ease of use, mainly due to the incomplete translation in Portuguese. Thus, there is a demand for new technologies that support nonlinear narratives creation.
\end{abstract}

Resumo. Narrativas não lineares permitem ao usuário a condução do fluxo da sua história, podendo percorrer caminhos diversos à medida que a história evolui, explorando diferentes níveis de lógica e criatividade. No entanto, as ferramentas digitais para essa criação são desconhecidas por muitos educadores. Por isso, este artigo tem como objetivo apresentar um mapeamento de trabalhos que exploram narrativas não-lineares no contexto brasileiro da Informática na Educação. O método adotado é o mapeamento sistemático, com inclusão de literatura cinzenta. Os resultados indicam que há um número pequeno de publicações sobre esse assunto. Além disso, existem limitações nas ferramentas existentes quanto à facilidade de uso, principalmente pela tradução incompleta em português. Logo, há demanda para novas tecnologias que auxiliem na criação de narrativas não-lineares.

\section{Introdução}

Como parte da evolução da humanidade, da simbologia à interlocução, a linguagem é fruto da capacidade de se comunicar e das interações do ser com o mundo à sua volta. A partir desta interlocução, nasce a capacidade de contar histórias, onde ficção, crença e narrativa são concebidas. Embora a maioria das produções disponíveis ao leitor sejam lineares, ou seja, narrativas com início, meio e um fim, um novo conceito narrativo tem se popularizado: as narrativas não-lineares. Esse tipo de narrativa constitui um método para contar histórias guiadas por meio de pontos de decisão, em que o leitor torna-se parte da evolução do conto. 
VIII Congresso Brasileiro de Informática na Educação (CBIE 2019)

Anais do XXV Workshop de Informática na Escola (WIE 2019)

Em meio educativo, a proposta tem promovido maior interação dos alunos com seus colegas e a própria narrativa, além de explorar habilidades como pensamento ação-reação, criatividade, entre outras. No entanto, considerando-se a exponencial complexidade que acompanha o fluxo constituinte desse tipo de história, o uso de ferramentas informatizadas podem facilitar esse processo.

Ao observar que as ferramentas existentes nesse contexto são desconhecidas por muitos educadores, este artigo tem como objetivo apresentar os recursos digitais disponíveis para criação de histórias não-lineares, a partir de uma pesquisa sistemática nas publicações da Comissão Especial de Informática na Educação (CEIE), bem como investigação na literatura cinzenta.

O artigo está organizado como segue. Na Seção 2, apresenta-se a conceituação de narrativas não-lineares. Na Seção 3, explica-se o método sistemático de pesquisa, bem como as questões de busca e palavras-chaves. Na Seção 4, descreve-se a síntese dos trabalhos encontrados sobre o tema. Por fim, a Seção 5 discorre as considerações finais.

\section{Narrativas não-lineares: diferentes percursos em uma mesma história}

Segundo McIntosh, Cohn e Grace (2010), as narrativas lineares seguem uma estrutura padronizada, começando pela exposição de um problema, que é explorado no meio e resolvido no final da história. A narrativa, bem como o ser, tem adquirido cada vez mais complexidade ao longo do tempo. A narrativa não-linear, por sua vez, foi disseminada por meio dos jogos. Nesse contexto, surgiram os chamados gamebooks, conhecidos no Brasil como livros-jogos, em que o leitor pode escolher os caminhos a serem percorridos. McIntosh, Cohn e Grace (2010) ressaltam que a chave para a narrativa não linear é a variabilidade. A Figura 1 exemplifica a diferença de fluxo entre as narrativas.
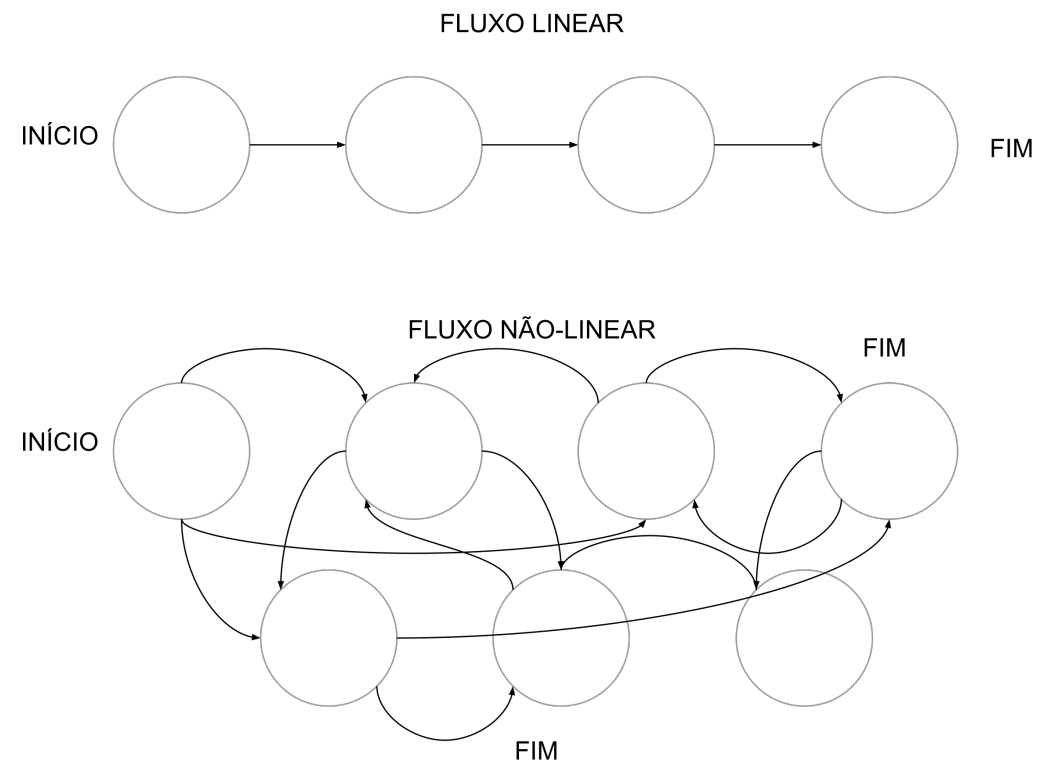

Figura 1. Exemplo de fluxos diferentes para narrativas lineares e não-lineares.

O aproveitamento dessas produções por profissionais da educação permite a elaboração de um material com maior capacidade de indução participativa e potencial multidisciplinar. É possível a realização de leitura personalizada pelo aluno, além da 
VIII Congresso Brasileiro de Informática na Educação (CBIE 2019)

Anais do XXV Workshop de Informática na Escola (WIE 2019)

reutilização da mesma obra com desfechos diferentes. Com isso, o potencial de interação e envolvimento com a narrativa é ampliado substancialmente. Além disso, a criação de narrativas não lineares contribuem para o pensamento ação-reação, criatividade, entre outras habilidades.

O problema é que criar narrativas com diferentes meios e vários finais possíveis pode ser uma tarefa demorada e complexa para organizar, principalmente no contexto da sala de aula. Por isso, o uso de ferramentas informatizadas pode facilitar esse processo. Nesse sentido, Sobreira, Nunes e Morassi (2013) descrevem uma abordagem de construção de narrativas não-lineares com 54 alunos do ensino fundamental. Os resultados indicam que a tecnologia mostrou-se uma forte aliada na integração de seus recursos para o alcance dos objetivos educacionais de forma prazerosa e criativa.

\section{O Processo de Mapeamento}

Segundo Nakagawa et al. (2017), Mapeamentos Sistemáticos (MS) são estudos secundários amplos sobre tópicos de pesquisa específicos, ou seja, o principal objetivo é identificar, avaliar e interpretar conteúdos relevantes a um tema, possibilitando o reconhecimento de lacunas para novas atividades de pesquisa.

O processo de um MS envolve três etapas principais: o planejamento, condução e publicação. Inicialmente é necessário identificar o objetivo do estudo e criar um protocolo de pesquisa, contendo as questões de busca, string utilizada nas fontes de consulta, além de critérios de inclusão e exclusão das publicações encontradas. Após, realiza-se a condução da busca e extração dos dados para posterior publicação, incluindo a devida discussão sobre os tópicos encontrados. A Figura 2 exemplifica o procedimento descrito.

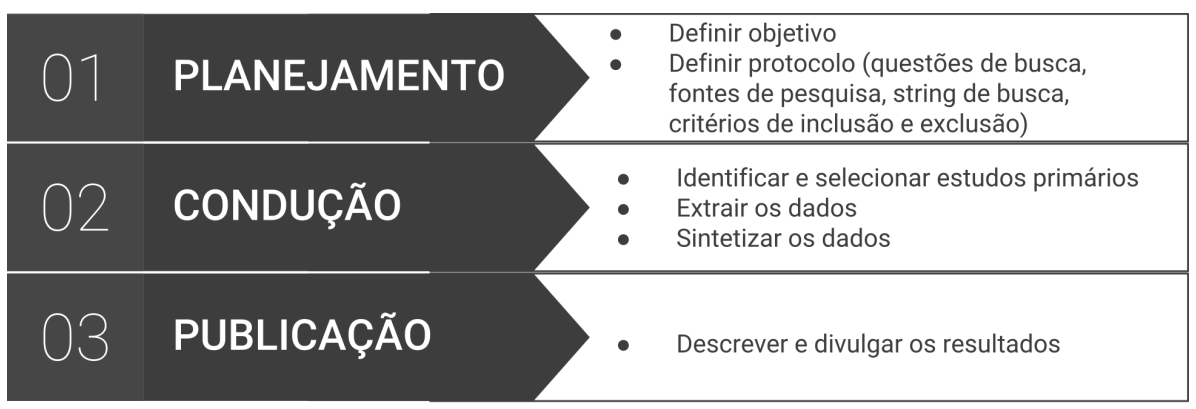

Figura 2. Fases para realização de um mapeamento sistemático: planejamento, condução e publicação (NAKAGAWA et al., 2017).

O objetivo deste MS é identificar ferramentas digitais que facilitem a criação de narrativas não-lineares no contexto da sala de aula. Após identificação da necessidade de realização do mapeamento, criou-se o protocolo com definição das questões de pesquisa, bases de consulta e string de busca, além dos critérios de inclusão e exclusão. Na sequência, realizou-se a fase de condução com a busca dos estudos primários, pré-seleção e extração de dados e por fim, a etapa de análise e síntese das informações. Quanto ao objetivo do mapeamento, este trabalho visa responder às seguintes Questões de Pesquisa (QP):

- QP1: Que recursos digitais existem para criação de narrativas não-lineares?

- QP2: Como a Informática na Educação explora as narrativas não-lineares no contexto brasileiro? 
VIII Congresso Brasileiro de Informática na Educação (CBIE 2019)

Anais do XXV Workshop de Informática na Escola (WIE 2019)

- QP3: Quais as características de uso das ferramentas existentes, como preço, idioma, requisitos de sistema operacional e funcionalidades?

Inicialmente, a base de consulta selecionada foi a da CEIE, que contempla publicações da Revista Brasileira de Informática na Educação (RBIE), do Simpósio Brasileiro de Informática na Educação (SBIE), Workshop de Informática na Escola (WIE), Jornada de Atualização de Informação na Educação (JAIE) e Anais dos Workshops do Congresso Brasileiro de Informática na Educação (CBIE) e do Workshop de Desafios da Computação Aplicada à Educação.

Conforme já descrito na Seção 2, a contação de histórias e livros-jogos estão diretamente relacionados ao tema. Logo, a seguinte string de busca foi usada: (não-linear AND narrativa) OR (não-linear AND história) OR (contação de histórias) OR (escolha AND história) OR (livro-jogo), bem como a tradução livre (nonlinear AND narrative) OR (nonlinear AND story) OR (storytelling) OR (choose and story) OR (gamebook). A mesma string traduzida em espanhol não retornou publicações.

Como critério de inclusão, definiu-se que seriam aceitos artigos independente do período de publicação, escritos ou traduzidos para os idiomas Português, Inglês ou Espanhol, pois são os idiomas aceitos para publicação na CEIE. Em relação ao critério de exclusão, em casos de duas ou mais publicações relativas ao mesmo trabalho, somente a publicação mais recente foi incluída na pesquisa. Assim, após definição dos parâmetros, iniciou-se a condução.

Apenas um artigo retornou com uso da string em português e outras sete publicações com uso da string em inglês. Dessa forma, notou-se a necessidade de ampliação do escopo de conteúdo. Como foram encontradas poucas referências a plataformas nacionais de criação de histórias não-lineares, observou-se a necessidade de aprofundamento, incluindo a literatura cinzenta. Gomes, Mendonça e Souza (2000) descreve que o termo literatura cinzenta é utilizado para denominar todas as referências não convencionais, produzidos no meio acadêmico, governamental e também pela indústria.

Logo, para explorar a literatura cinzenta adotou-se a busca pelas palavras-chaves "tools narrative nonlinear" e suas respectivas traduções em português, no Google. Os resultados obtidos na extração de dados são descritos a seguir.

\section{Resultados e Discussão}

Esta Seção apresenta a súmula dos principais resultados encontrados na busca por publicações no contexto de narrativas não-lineares, além de ferramentas digitais que apoiem esse processo.

\subsection{Publicações da CEIE}

A pesquisa na base da CEIE retornou 7 artigos, apresentados na Tabela 1. Percebese que a maioria dos resultados retornados são publicações do WIE (5), seguidos de artigos dos workshops do CBIE (2) e um artigo do SBIE.

O trabalho de Rosa et al. (2017) trata de um projeto que combina o uso de um simulador (OpenSim) com um serviço de bots on-line (PandoraBots). Esses recursos são usados para os alunos assistirem a uma peça de teatro digitalmente, no contexto de ensino de teste de software. 
VIII Congresso Brasileiro de Informática na Educação (CBIE 2019)

Anais do XXV Workshop de Informática na Escola (WIE 2019)

Tabela 1. Publicações resultantes da busca na base da CEIE.

\begin{tabular}{|l|l|l|}
\hline Título & Autores & Local \\
\hline $\begin{array}{l}\text { Ensino de testes de software por meio de digital Story- } \\
\text { telling e Chatterbots }\end{array}$ & Rosa et al. (2017) & SBIE \\
\hline $\begin{array}{l}\text { A utilização do laptop educacional na contação de } \\
\text { história }\end{array}$ & $\begin{array}{l}\text { Melo, Bezerra e Filho } \\
(2015)\end{array}$ & WIE \\
\hline $\begin{array}{l}\text { Aligning Digital Storytelling to the TPACK Framework: } \\
\text { A Learning Experience for Pre-Service Teachers in a } \\
\text { Learning-by-Designing Project }\end{array}$ & $\begin{array}{l}\text { Harriman e Branch } \\
\text { (2012) }\end{array}$ & WIE \\
\hline $\begin{array}{l}\text { Aplicando o group storytelling no compartilhamento de } \\
\text { experiências e na avaliação em sala de aula }\end{array}$ & Lage et al. (2017) & WIE \\
\hline $\begin{array}{l}\text { Framework de ensino de programação para crianças e } \\
\text { jovens por meio de aprendizado baseado em projetos } \\
\text { usando computação tangível, storytelling, internet das } \\
\text { coisas e sistemas embarcados }\end{array}$ & Leite e Branco (2017) & CBIE \\
\hline $\begin{array}{l}\text { Pensamento Computacional: Uma Proposta de Ensino } \\
\text { com Estratégias Diversificadas para Crianças do Ensino } \\
\text { Fundamental }\end{array}$ & Reis et al. (2017) & WIE \\
\hline Banda da Floresta & Carneiro e Silva (2016) & CBIE \\
\hline $\begin{array}{l}\text { Produzindo histórias não lineares: um incentivo à } \\
\text { produção escrita e leitura, através do uso contextuali- } \\
\text { zado da tecnologia }\end{array}$ & $\begin{array}{l}\text { Sobreira, Nunes e Mo- } \\
\text { rassi (2013) }\end{array}$ & WIE \\
\hline
\end{tabular}

Melo, Bezerra e Filho (2015) relatam o uso dos programas KWord, TuxPaint e Google Drive como apoiadores para a produção de histórias. O KWord foi utilizado para edição de texto, o TuxPaint para criação de desenho e a produção colaborativa on-line foi apoiada pelo Google Drive. Embora o foco tenha sido a escrita de histórias, salienta-se que as narrativas criadas não tinham caráter não-linear.

O estudo de Harriman e Branch (2012) tem como objetivo promover uma avaliação a professores que usam ferramentas de narrativa. As ferramentas citadas são: Storybird, VoiceThread, PhotoStory e iMovie. Storybird é um site para criação de histórias lineares ilustradas. VoiceThread combina um software de apresentação de slides com uma gravação em áudio ou texto sobre ele, voltado para apresentações on-line. PhotoStory e iMovie são softwares de edição de vídeo. As ferramentas utilizadas exploram apenas o contexto linear para criação de histórias.

O objetivo do trabalho de Lage et al. (2017) foi construir histórias contadas em grupo como forma de avaliação de uma disciplina. São utilizadas duas ferramentas: CMap e Sofia 2.0. Sofia 2.0 é um organizador de fragmentos de uma história maior. Ainda que a ferramenta permita a criação de histórias interativas, a leitura exige interação de um grupo para leitura. Por não estar disponível para o público geral, não foi possível avaliar a ferramenta. Ainda assim, por meio do artigo foi possível aferir a considerável curva de aprendizado necessária para a utilização plena da plataforma, o que levou os alunos do estudo a migrarem para a outra ferramenta, CMap (foco em criação de mapas mentais).

Leite e Branco (2017) descrevem um framework para ensino de programação a crianças que engloba diversas atividades, entre elas, storytelling. No mesmo sentido, Reis 
VIII Congresso Brasileiro de Informática na Educação (CBIE 2019)

Anais do XXV Workshop de Informática na Escola (WIE 2019)

et al. (2017) relatam uma atividade com foco principal em ensino de programação. Já Carneiro e Silva (2016) apresenta um aplicativo com uso de realidade aumentada para crianças. Porém, nenhum dos trabalhos apresentados citam narrativas não-lineares.

Por fim, Sobreira, Nunes e Morassi (2013) apresentam a única produção alinhada aos objetivos desta pesquisa. Os autores propõem a construção de uma história não-linear com uso de recursos tecnológicos. As ferramentas citadas são o Gimp, para criação de desenhos e editor de apresentação de slides com auxílio de links para viabilidade dos diferentes percursos na história. Além disso, utilizou iSpring para conversão em formato flash e publicação na internet.

Os resultados apresentados respondem à QP2, ou seja, narrativas não-lineares ainda é um tema pouco discutido no âmbito da Informática na Educação Brasileira, visto que foi encontrado apenas um relato que explora o tipo de história e que não utiliza ferramenta específica para facilitar a criação. Dessa forma, a seguir são apresentadas ferramentas já disponibilizadas na indústria para uso nesse contexto, a fim de responder as questões QP1 e QP3 .

\subsection{Literatura Cinzenta}

Conforme já descrito, houve necessidade de uma busca ampliada para análise de ferramentas digitais que apoiam a criação de narrativas não-lineares. A investigação dos resultados teve o intuito de conhecer e explorar as ferramentas gratuitas disponíveis, além de elencar facilidades e limitações dessas plataformas. Foram encontradas 11 ferramentas, sendo 10 totalmente gratuitas.

Adrift (2018) permite a criação de vários mapas para uma mesma história e visualização em 3 dimensões das localizações. Não exige conhecimento em nenhum tipo de linguagem específica, a construção acontece através do preenchimento de campos descritivos. Já a ChoiceScript (2018) é uma linguagem de programação para escrever jogos de múltipla escolha, possibilitando criação de histórias não-lineares. Porém, a edição é off-line e apesar de permitir edição em qualquer sistema, a organização é complexa e depende da localização relativa dos arquivos.

Inform (2018) é um programa para a produção de narrativas textuais com escrita direta. Algumas palavras e estruturas de frases atuam como a linguagem de definição e marcação do programa. Uma interface gráfica está disponível para melhor visualização do trabalho escrito. Já a Ren'Py é uma plataforma open-source gratuita para uso comercial que exige conhecimento prévio de lógica de programação e da linguagem de programação Python. Em sua gama de ferramentas, o programador dispõe de transições, animações e outros componentes visuais para sua produção. Além disso, o trabalho criado pode ser executado em sistemas operacionais desktop e mobile (REN'PY, 2018).

O Text Adventure Development System (TADS, 2018) é um compilador de narrativas textuais escrita na própria plataforma através de linguagem de programação. Por ser uma das primeiras plataformas do gênero, é frequentemente citado por outras iniciativas. Requer instalação desktop para criação e execução das histórias. Caso haja um servidor com TADS, é possível a execução remota através de um navegador.

Alguns recursos interessantes estão disponíveis no portal Text Adventures ${ }^{1}$, como

\footnotetext{
${ }^{1}$ Disponível em 〈http://textadventures.co.uk/〉
} 
VIII Congresso Brasileiro de Informática na Educação (CBIE 2019)

Anais do XXV Workshop de Informática na Escola (WIE 2019)

as ferramentas Quest e Squiffy. O Quest permite a criação de dois tipos de história: a narrativa textual e o livro jogo. Por meio de uma interface gráfica os detalhes de cada cena (narrativa textual) ou página (livro jogo) são preenchidos em formulários para a construção geral da história. A maior limitação é executar apenas em computadores com Windows ou no site da plataforma (ADVENTURES, 2018a).

O Squiffy também permite a criação de livro-jogo, mas sua diferença se encontra na forma em que se escreve a história, exigindo linguagem de marcação para determinar as ligações entre páginas e JavaScript para execução de recursos mais avançados. A vantagem é permitir o download dos arquivos da história, garantindo execução em qualquer sistema com um navegador web (ADVENTURES, 2018b).

A plataforma Infinite Story (2018) proporciona uma experiência on-line de simples navegação, em que a criação da história é feita por meio de respostas a formulários estruturados com os campos: room, choices, image, image location e room nickame. Esses campos servem para inserir a história, as alternativas que o usuário poderá escolher, imagem de passagem, imagem no bloco e um apelido para a cena, respectivamente. Com isso, não é necessário ser feita a conexão entre os quadros, pois, através do preenchimento dos formulários, a história é estruturada naturalmente.

Millington (2018) é um conjunto de arquivos nos padrões web (HTML, JS e CSS) que permite a edição das histórias direto nos arquivos base. As funções permitem construir livros-jogo que registram e consultam dados de páginas visitadas anteriormente, o que reduz a repetição de conteúdo. Os arquivos podem ser visualizados em qualquer navegador moderno.

Uma plataforma citada em alguns trabalhos é a Twine (2018). A ferramenta apresenta uma camada de interface gráfica bastante simples e intuitiva. A construção da narrativa é feita por blocos que são apresentados na tela e podem ser conectados uns aos outros por meio de linhas. Porém, para fazer a ligação entre as opções de decisão de cada bloco, é necessária a utilização de linguagem de marcação HTML. Esta, embora simples, não é explicitamente informada em tela, podendo deixar o usuário perdido em um primeiro contato. A tradução para português é apenas parcial. A Tabela 2 apresenta uma síntese das ferramentas encontradas no estudo, salientando as principais características. Além do idioma, é apresentado o modo de criação de narrativas nas ferramentas e se possibilitam galeria própria para publicação das produções.

Das ferramentas encontradas, a TILT (2018) é a única plataforma brasileira. Embora seja paga, possibilita a criação de narrativas não-lineares na ferramenta chamada MAscript, com limite na quantidade de objetos (versão de teste). A plataforma utiliza conjuntos de códigos de programação para a construção das histórias, mas nem todas as palavras reservadas estão em português. Qualquer navegador moderno é capaz de apresentar as narrativas.

Das plataformas exploradas, apenas duas apresentam versão em português, uma em processo de tradução e outra que requer assinatura para acesso a todas as funções. A maioria das ferramentas permite a leitura das histórias em navegadores web, propiciando maior flexibilidade de sistemas operacionais e até mesmo a utilização de dispositivos móveis. Além disso, é possível adicionar conteúdo multimídia, como figuras e animações.

O Twine foi a única ferramenta que permite a criação de histórias totalmente por 
VIII Congresso Brasileiro de Informática na Educação (CBIE 2019)

Anais do XXV Workshop de Informática na Escola (WIE 2019)

Tabela 2. Síntese de Características das Ferramentas

\begin{tabular}{|l|l|l|l|l|}
\hline Ferramenta & Idioma & Plataforma & $\begin{array}{l}\text { Modo de } \\
\text { Criação }\end{array}$ & Galeria \\
\hline Adrift & Inglês & Windows & formulário & X \\
\hline Choice Script & Inglês & Multiplataforma (off-line) & script & - \\
\hline Inform & Inglês & Multiplataforma (off-line) & script & - \\
\hline Ren'Py & Inglês & Multiplataforma (off-line) & script & X \\
\hline TADS & Inglês & Multiplataforma (off-line) & script & - \\
\hline Quest & Inglês & Windows e On-line & formulário & X \\
\hline Squiffy & Inglês & Multiplataforma (on-line) & script & X \\
\hline Infinite Story & Inglês & On-line & formulário & X \\
\hline Undum & Inglês & Multiplataforma (off-line) & script & - \\
\hline Twine & 13 idiomas + & Multiplataforma (on-line) & interface gráfica & X \\
\hline TILT & Português & Pcript & X \\
\hline
\end{tabular}

meio de interface gráfica, as demais exigem conhecimentos específicos de linguagens de marcação e programação. Apenas o Inform não requer nenhum tipo de linguagem de programação para utilizar todos seus recursos, apenas escrita comum. Entretanto, há a palavras em inglês reservadas para realizar as marcações das opções da história, o que pode confundir o usuário.

Todas as plataformas avaliadas são gratuitas, entretanto a TILT requer uma assinatura anual para o uso ilimitado de todos os recursos disponíveis da plataforma. Quest e Squiffy pertencem ao mesmo grupo, e possuem o serviço gratuito ActiveLit. A maioria possui algum tipo de portal on-line, onde é possível compartilhar as histórias online e até monetizar, servindo como incentivo para os usuários finalizarem seus trabalhos. Com o ActiveLit, é possível o gerenciamento das histórias em grupos de alunos organizados por turmas. A próxima Seção discute os resultados apresentados neste estudo.

\section{Considerações Finais}

Observa-se que se faz oportuno o uso de ferramentas computacionais que forneçam um ambiente facilitador de criação e leitura de histórias não-lineares. Logo, o presente estudo teve como objetivo pesquisar e analisar recursos digitais existentes para a criação de narrativas não-lineares, além de identificar como a área de Informática na Educação explora esse tipo de narrativa.

Inicialmente, realizou-se um mapeamento na base da CEIE e percebeu-se que sete trabalhos citam uso de narrativas, porém somente um estava alinhado aos interesses desta pesquisa. Dessa forma, ampliou-se a busca, incluindo software da indústria e demais produtos considerados literatura cinzenta. Esse resultado mostra que há um espaço para investigação dentro da área, alinhando narrativas não-lineares e informática. Além da possibilidade de criação de ferramentas digitais que incentivem essas produções, relatos de experiência da aplicação das ferramentas existentes em sala de aula podem ser práticas motivadoras a professores da educação básica, principalmente para inclusão do computador em atividades de leitura e escrita, de forma dinâmica e divertida. 
VIII Congresso Brasileiro de Informática na Educação (CBIE 2019)

Anais do XXV Workshop de Informática na Escola (WIE 2019)

Quanto às plataformas existentes, percebem-se limitações de uso para sua popularização em atividades de ensino. A maioria das ferramentas analisadas exigem que o usuário conheça pelo menos alguma linguagem de marcação, e os programas mais avançadas requerem conhecimento em linguagem e lógica de programação. Por se tratar de um objetivo muito específico, algumas plataformas criam personalizações de linguagens de programação já existentes - o que cria mais possibilidades, mas afasta usuários sem conhecimento prévio em programação.

A maioria das plataformas possibilita a execução em qualquer sistema operacional para desktop. Algumas permitem apenas o uso no navegador, um fator limitante à escolas que não tenham conexão com internet. Salienta-se também como déficit a falta de responsividade das ferramentas on-line, reduzindo possibilidade de uso em tablets e smartphones. Logo, considera-se um desafio a ser pensado nos grupos de pesquisa aplicada, como prover a criação de narrativas não-lineares em dispositivos móveis.

Como ameaças à validade do estudo, reconhece-se que a inclusão de bases internacionais possibilitaria uma análise do envolvimento da comunidade acadêmica em torno das plataformas de narrativas não-lineares, porém o escopo deste mapeamento tinha como foco os estudos desenvolvidos no país. Além disso, apesar de contemplar os principais meios de publicação da área (CEIE), outras bases de trabalhos nacionais podem conter artigos relacionados.

Por fim, considera-se que os prováveis motivos para escassez dessas produções no Brasil podem ser a falta de plataformas em português e o fato dos professores não dominarem habilidades de programação, um dos fatores limitantes dos recursos encontrados. Assim sendo, fica clara a oportunidade de desenvolvimento de uma aplicação de código livre em português, que dê preferência por métodos mais intuitivos para criação de histórias não-lineares.

\section{Referências}

ADRIFT. ADRIFT Adventure Development \& Runner - Interactive Fiction Toolkit. 2018. Disponível em: 〈http://www.adrift.co/new/〉. Acesso em: 12 jun. 2018.

ADVENTURES, T. Quest - Build text adventure games and interactive fiction. 2018. Disponível em: 〈http://textadventures.co.uk/quest〉. Acesso em: 12 jun. 2018.

ADVENTURES, T. Squiffy - A simple way to write interactive fiction. 2018. Disponível em: 〈http://textadventures.co.uk/squiffy . Acesso em: 12 jun. 2018.

CARNEIRO, R.; SILVA, R. T. da. Banda da floresta. In: Anais dos Workshops do Congresso Brasileiro de Informática na Educação. [S.1.: s.n.], 2016. v. 5, n. 1, p. 112.

ChoiceScript. 2018. Disponível em: 〈https://www.choiceofgames.com/ make-your-own-games/choicescript-intro//. Acesso em: 12 jun. 2018.

GOMES, S. L. R.; MENDONÇA, M. A. R.; SOUZA, C. d. Literatura cinzenta. Fontes de informação para pesquisadores e profissionais, UFMG Belo Horizonte, p. 97-citation_lastpage, 2000.

HARRIMAN, C. L. S.; BRANCH, R. M. Aligning digital storytelling to the tpack framework: A learning experience for pre-service teachers in a learning-by-designing 
VIII Congresso Brasileiro de Informática na Educação (CBIE 2019)

Anais do XXV Workshop de Informática na Escola (WIE 2019)

project. In: Anais do Workshop de Informática na Escola. [S.1.: s.n.], 2012. v. 1, n. 1.

Infinite Story. Infinite Story - Interactive Fiction Engine. 2018. Disponível em: 〈https://infinite-story.com〉. Acesso em: 12 jun. 2018.

INFORM. Inform 7. 2018. Disponível em: 〈http://inform7.com〉. Acesso em: 12 jun. 2018.

LAGE, B. et al. Aplicando o group storytelling no compartilhamento de experiências e na avaliação em sala de aula. In: Anais do Workshop de Informática na Escola. [S.1.: s.n.], 2017. v. 23, n. 1, p. 165.

LEITE, K.; BRANCO, K. C. Framework de ensino de programacão para crianças e jovens por meio de aprendizado baseado em projetos usando computação tangível, storytelling, internet das coisas e sistemas embarcados. In: Anais dos Workshops do Congresso Brasileiro de Informática na Educação. [S.1.: s.n.], 2017. v. 6, n. 1, p. 1301.

MCINTOSH, B.; COHN, R.; GRACE, L. Nonlinear Narrative in Games: Theory and Practice. 2010. Disponível em: 〈https://www.gamecareerguide.com/features/882/features/ 882/nonlinear \_narrative $\backslash$ in $\backslash$ games $\backslash$ _.php $\rangle$. Acesso em: 1 jul. 2018.

MELO, M. B. O. de; BEZERRA, E.; FILHO, J. A. de C. A utilização do laptop educacional na contação de história. In: Anais do Workshop de Informática na Escola. [S.1.: s.n.], 2015. v. 21, n. 1, p. 310.

MILLINGTON, I. Undum. 2018. Disponível em: 〈https://github.com/idmillington/undum $\rangle$. Acesso em: 12 jun. 2018.

NAKAGAWA, E. et al. Revisão Sistemática da Literatura em Engenharia de Software: Teoria e Prática. Elsevier Editora Ltda., 2017. ISBN 9788535285970. Disponível em: $\langle$ https://books.google.com.br/books?id=kCspDwAAQBAJ $\rangle$.

REIS, F. de M. et al. Pensamento computacional: Uma proposta de ensino com estratégias diversificadas para crianças do ensino fundamental. In: Anais do Workshop de Informática na Escola. [S.1.: s.n.], 2017. v. 23, n. 1, p. 638.

REN'PY. RenPy What is RenPy. 2018. Disponível em: 〈https://www.renpy.org 〉. Acesso em: 12 jun. 2018.

ROSA, L. H. C. et al. Ensino de testes de software por meio de digital storytelling e chatterbots. In: Brazilian Symposium on Computers in Education (Simpósio Brasileiro de Informática na Educação-SBIE). [S.1.: s.n.], 2017. v. 28, n. 1, p. 797.

SOBREIRA, E. S. R.; NUNES, M. C.; MORASSI, S. B. Produzindo histórias não lineares: um incentivo à produção escrita e leitura, através do uso contextualizado da tecnologia. In: Anais do Workshop de Informática na Escola. [S.1.: s.n.], 2013. v. 1, n. 1, p. 160.

TADS. TADS - the Text Adventure Development System. 2018. Disponível em: $\langle$ http://www.tads.org $\rangle$. Acesso em: 12 jun. 2018.

TILT. TILT - MicroAdventures. 2018. Disponível em: 〈http://tilt.net/ztilt/index.php〉. Acesso em: 12 jun. 2018.

Twine. 2018. Disponível em: 〈http://twinery.org〉. Acesso em: 12 jun. 2018. 